\section{Sénécal, Gilles et Laurence Bhérer (dir.) La métropolitisation et ses territoires, Québec, Presses de l'Université du Québec, Collection Science régionale, 2009, 291 p.}

Voilà un ouvrage qui va intéresser ceux qui aiment détester Montréal, qu'ils y habitent ou qu'ils soient en régions centrales ou périphériques, peu importe. Les premiers ont eu l'occasion de s'exprimer ${ }^{1}$ récemment lors des élections municipales marquées par les scandales reliés à la corruption. Cependant, il n'est nullement question de corruption ici. On a l'impression que, tout comme le maire réélu, aucun des auteurs, au moment de la rédaction de l'ouvrage, était au courant de qui a fait coulé tant d'encre à l'automne 2009. La majorité des 21 collaborateurs à cet ouvrage, Québécois pour la grande majorité, ont porté une attention à d'autres problèmes qui affectent notre chère métropole. Le tout débute par une citation en exergue de Flora Tristan rendue célèbre par ses Promenades dans Londres (1839). Cette féministe avant la lettre considérait la capitale de la fière Albion trop grande et trop étendue pour se fréquenter ou se connaître. Que dirait-elle aujourd'hui de Montréal, une ville dont il est surtout question dans ce volume? Ce n'est pas l'étendue qui pose problème à la métropole québécoise aux yeux de certains auteurs mais plutôt, et avec, ô combien raison, son mode de gouvernance on ne peut plus chaotique et marqué par un immobilisme qui la fait bien mal vieillir en comparaison avec des villes américaines comme Boston, Chicago, Philadelphie et autres Seattle ou encore des villes françaises telles Paris, Lyon, Montpellier, Bordeaux, Dijon et Toulouse pour n'en nommer que quelques-unes.

Pour les responsables de l'ouvrage, en plus de la question de la gouvernance, la métropolisation soulève celle des environnements naturels et construits en faisant un enjeu pour la qualité de vie et, par delà, pour la compétitivité économique. Les différents collaborateurs s'interrogent sur la construction de l'objet social qu'est la métropole, de façon générale, ainsi que sur les différentes facettes de la légitimisation de ses territoires et des échelles appropriées d'analyse du fait urbain. Ainsi, le regretté C. Manzagol de l'Université de Montréal, à la faveur d'une revue de la littérature récente, évoque avec nostalgie l'ancienne organisation économique et sociale marquée par ce que Durkheim qualifiait de «solidarité organique ». Aujourd'hui, avec la montée de l'individualisme et de la mobilité, on se trouve aux prises avec l'affaiblissement de ce lien social autrefois si présent. Si cette caractéristique n'est évidemment pas propre à Montréal. C'est à cette dernière que se consacre, comme la plupart des auteurs, le duo AlvergneLatouche. La première, familière des lecteurs de cette chronique, épouse du second, était, au moment d'écrire ses lignes, à l'emploi de l'ONU à Dakar, comme conseillère en développement local. Le compte-rendu qui accompagne celui-ci est le troisième que j'ai eu le plaisir de rédiger sur ses écrits pour cette chronique. Quant à monsieur, heureux retraité de l'INRSUrbanisation Culture et Société, il s'affaire comme consultant en Afrique.

Le tableau présenté n'est pas, beaucoup s'en faudrait, des plus optimistes. S'ils soulignent que la ville, dite autrefois aux cent clochers ${ }^{2}$ occupe le quatrième rang en Amérique du Nord pour le nombre d'emplois de haute technologie, le même rang pour la sécurité des rues, qu'elle produit près de 500 doctorats dans tous les domaines, qu'elle se classe au $7^{\mathrm{e}}, 10^{\mathrm{e}}$ et $13^{\mathrm{e}}$ rangs dans les indices de Richard Florida se rapportant à la tolérance (oui, on y est si accommodants...), au talent (le fameux indice bobo : bourgeois-bohème) et à la technologie. Après avoir lancé d'autres fleurs du genre, le pot ne tarde pas à suivre. Montréal est une ville pauvre : au dernier rang de la vingtaine de villes nord-américaines auxquelles on la compare habituellement. Avec $13 \%$ de riches, Montréal se situe derrière des villes comme Rimouski, Cowansville et même Gaspé. Grâce à ce constat, les auteurs ne voient comment on peut conclure que la ville aux trop nombreux roitelets se serait enrichie sur le dos des régionaux. Que ces derniers se le tiennent pour dit... Pour ce qui est de la création d'entreprises, la situation, selon notre couple franco-québécois, à l'image de celle du Québec, serait pitoyable. Si la ville se caractérise par un nombre élevé de personnes au travail, leur productivité (en dehors du secteur de haute technologie) est médiocre. Ces problèmes sont connus et on en discute. 
C'est ce que montre Claire Poitras, toujours active à l'INRS-Urbanisation, en faisant allusion aux acteurs ayant participé à la promotion et à l'évaluation de deux projets se rapportant à des aménagements autoroutiers dans la région de Montréal. L'auteure dégage les arguments tirés des représentations divergentes (on l'aurait deviné). Et, c'est en vertu de ces divergences que rien ne se fait pour le plus grand bonheur des CAVES (Citezens Againts Virtualy Everything) très nombreux à Montréal, tel que je l'ai déjà signalé. Ce n'est pas à eux que pense (contrairement à l'auteur de ces lignes) JeanMarc Fontan, un auteur familier aux lecteurs d'O\&T. En collaboration avec trois autres auteurs dont P. Hamel de l'Université de Montréal, mon collègue de l'UQAM traite de l'Empowerment culturel, social, politique et économique de populations à la marge de l'économie mondiale. Il s'agit d'individus œuvrant au sein d'organismes communautaires susceptibles d'exercer une influence à l'intérieur des mécanismes de gouvernance locale. Or, de leurs travaux, il se dégage que les acteurs de la société civile restent confinés à l'action locale sans exercer une influence sur la croissance économique et la compétitivité à l'échelle internationale. Il semble qu'il fallait faire une recherche (bien subventionnée) pour aboutir à une telle évidence... Une situation que déplorent mes collègues qui me paraissent accrochés à la fameuse devise de Baden Powel : Scout un jour, scout toujours. Peuvent toujours rêver. Faut-il le répéter, le développement économique ne se fait pas à la faveur de décisions prises à mains levées dans un sous-sol d'église (encore ouverte).

Un peu dans la foulée de ce chapitre sur la participation citoyenne et les illusions qui s'y rattachent, Luc Ducasse de l'UQAM, en traitant surtout des corporations de développement économique et communautaire (CDEC), se demande si le loup n'est pas dans la bergerie. Oui, pôvres moutons! Comme les CDEC ont vu leur marge de manoeuvre se rétrécir considérablement ces dernières années, mon collègue uqamien vise ici à jeter une lumière nouvelle sur les canaux par lesquels une approche plus bureaucratique s'est vue imposée aux CDEC par l'État (Ottawa ou Québec?) tout en cherchant à voir comment les élus municipaux et les fonctionnaires s'immiscent dans leur processus décisionnel.

Dans un épilogue, J. A. Bouderau et J. P. Colin également de l'INRS-Urbanisation, culture et société, font remarquer que, si à Montréal, le référentiel néolibéral du New Regionalism était présent au milieu des années 1980, les mécanismes de gouvernance métropolitaine qui s'y rattachent ont laissé place aux mécanismes d'institutionnalisation classique. Alors qu'à Toronto, la pensée métropolitaine demeure toujours bien ancrée dans l'esprit de ce New Regionalism. Dans ce que l'on désigne comme étant la ville Reine, les interventions d'acteurs de la société civile, de l'élite économique, d'un maire proactif (que les montréalais aimeraient bien avoir), de fonctionnaires provinciaux et municipaux contribuent à privilégier des outils territoriaux novateurs. Le Grand Paris se donne également en exemple à la suite de la mise en place de mécanismes de gouvernance novateurs caractérisés par une certaine souplesse territoriale. Malgré la grande centralisation de l'État français (le fameux mal français), les auteurs, dans les dernière pages du volume, signalent que tous les espoirs sont permis pour voir émerger à Paris un espace métropolitain délibératif. En conséquence, si c'est vrai qu'en se regardant on se désole et en se comparant on se console, le lecteur parisien pourrait lui aussi tirer profit de la lecture de cet ouvrage qui donne une idée de l'univers dans lequel doivent se dépêtrer leurs semblables quelque part outre-mer. Mais, bien entendu, c'est au lecteur québécois avant tout que cet ouvrage se destine.

\footnotetext{
Notes

${ }^{1}$ Beaucoup ne l'ont pas fait étant donné le très faible taux de participation.

${ }^{2}$ En référence au grand nombre d'églises au temps de mon enfance. Aujourd'hui, elles ferment toutes les une après les autres. Il serait plus pertinent de parler de la ville aux cent terrains de stationnement à ciel ouvert pour désigner une ville qui a du mal à remplacer les vieux édifices démolis.
} 


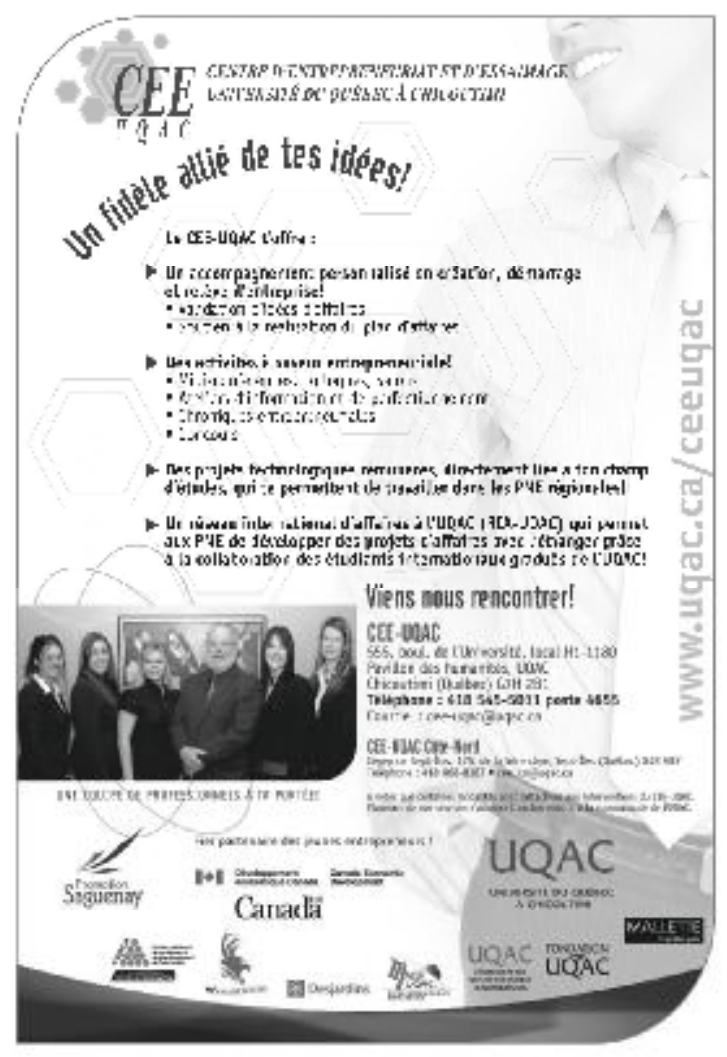

\section{Programme de Maitrise}

\section{B A POUR CADRES}

Un protgamme adapté aux tadte en exercicx.

La personnte cadre développe ses qualités

de gestionngire par l'étude de ass cancrelds at la travail en équipe, co qui lui permet d’älabarar

de nouvelles stratégies do is le contexte

de la fluctuation ox de la mondialisation der manchá.

\section{Conditions dadmission}

Posséder quatre années d'expérience comme cadre et un baccalauréat en gestion ou une maitrise ou une expérience professionnelle exceptionnelle.

\section{UQP}

\section{Thierno Diallo}

Dép. des sciences économiques et administratives 555, boul de l'Universite, Chicoutimi fOuebect G7H 281

Tel. : 418 545-5011, poste 5282. Télec.: $418693-9072$

Courriel : Thierno.Disllo@uqac.cs www.uqae.es

\section{Maitrise en gestion \\ des organisations}

\section{LES DÉrAS}

- Créer de nouvelles entreprises

- Affronter une concurrence mondiale

- Innover pour améliorer la performance organisationnelle

\section{LE PROGRAMME}

Cinq orientations : Entrepreneurship, international et innovation, développement organisationnel et éthique de l'organisation

Deux profils : Professionnel (stage) et scientifique (mérnoire)

LACDNTĖE La Maitrise en gestion des arganisations s'adresse aux gens désireux de développer leurs connaiseances ot habiletís afin de devenit acteurs du changement et permettre a leur organisstion de se developper. Renselgnements supplémentaires 545-5011, poste 5282

Formulaires de demande d'admission disponibles en vous adressant au secrétariat de la Maitrise ou au Bureau du registraire.

\section{UQAC}

555, boul. de I'Université, Chicoutimi (Québec) G7H 2B1 Tól.: 418 545-5011, poste 5282. Télec: $418693-9072$ www.uqac.ca

\section{Baccalauréat} en administration

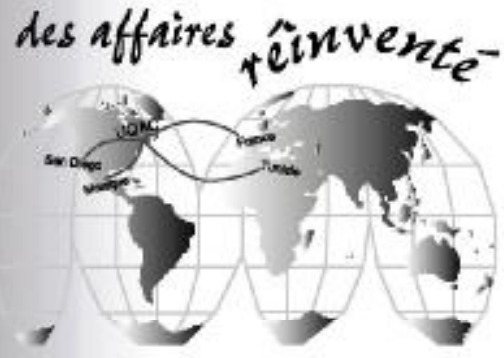

Découvrez le monde avec nous

Démystifiez la mondialisation Apprivoisez la diversité culturelle

Visez I'excellence, relevez vos défis avec le nouveau BAA

Obtenez simultanément le Diplôme de BAA et celui de l'Université

de San Diego en Californie (une première au Canada et au Québec)

\section{UQAC}

UNTEEsite du Qufaec A CHICOUmMI

Module des sciences de l'administration

555, boul. de FUniversite, Chicoutimi (Québoc) G7H 281 Tol. : 4185455011 , poste 5292. TUloc: 4186939072 www.uqac.ca 


\section{Innover c'est bien... breveter c'est mieux!}

Comment faire de l'argent avec des brevets 


\section{CONTENU DU LIVRE}

\section{SECTION 1 : LE BREVET, MYTHES ET RÉALITÉS}

Cette première section du livre est consacrée à l'exploration des différents mythes populaires liés à la notion de brevet :

- Est-ce que par l'acquisition d'un brevet, un entrepreneur se protège de mises en demeure liées à son produit?

- Est-ce vrai qu'un brevet coûte cher et qu'il ne rapporte aucun revenu?

- Comment défendre un brevet lorsqu'il est copié ?

- Est-ce qu'un entrepreneur a les moyens financiers pour défendre son brevet?

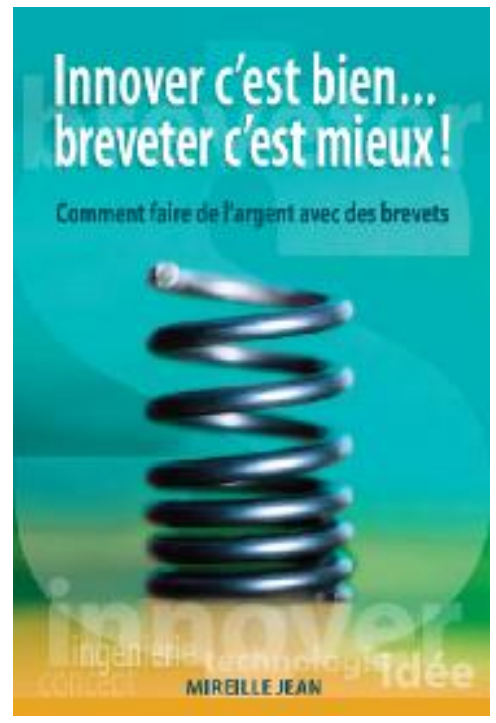

\section{SECTION 2 : COMMENT FAIRE DE L'ARGENT AVEC UN BREVET ?}

Cette section démontre comment il est possible de faire de l'argent avec l'acquisition de brevets. Ces chapitres constituent des pistes de réflexion pour faire fructifier les investissements.

- Pourquoi une innovation brevetée peut générer plus de revenus qu'une innovation non brevetée ?

- Quels sont les quatre moyens accessibles à l'entrepreneur pour faire de l'argent ? Est-il mieux de miser sur un produit exclusif, une licence, la vente de son brevet ou miser sur la contrefaçon ?

De plus, Mme Jean démontre par différents calculs simples, comment évaluer rapidement la valeur financière d'un brevet. Cette méthode d'évaluation permettra à l'entrepreneur de chiffrer son innovation, mais aussi de se bâtir une argumentation efficace.

\section{SECTION 3 : TRUCS ET ASTUCES POUR LA PME}

Cette section, est le «coffre à outils » des entrepreneurs en matière de brevet.

- Quels sont les risques d'invalidation d'un brevet?

- Par qui et comment un brevet peut être contrefait ?

- Comment se défendre face à la contrefaçon ?

- Comment tirer le maximum de la licence ?

- Quand doit-on choisir un agent de brevets et un avocat ? 


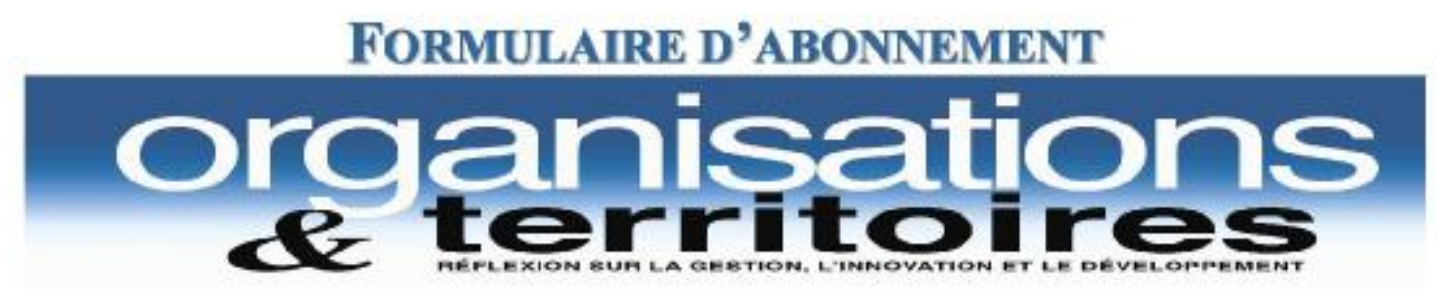

Je désire $m$ 'abonner à la revue Organisatüs et territaires at je jains un chèque av montant de :

Nouvel abonné $(\hat{\gamma})$ :

Renouvellement $(\sqrt{ })$ :

Tate:

Abonncment institutionnel (6 numcros) $101,59 \$$ (Toutes taxes incluses)

Abonnement individuel (6 numéros) 65,47\$ (Toutcs taxcs incluses)

Abonnement ćudjan. (6 numcros) 47,41\$ (Tx inc.) (Envoyer photocopie carte étud.)

Abonnement gouvernemental (6 numéros) $90,00 \$ \quad$ (Fournir certificat d'exemption des taxes)

Abonnement hors Canada (6 numéros) 145,00 $\$ \quad(110 \$+35 \$$ frais de poste) (sans taxes)

Tous les abonnements sont d'une durée de deux ans

- J'aimerais que mon abonnement débute avec le DERNIER numéro paru. soit :

Volume $\mathrm{N}^{\circ}$

- J'aimerais que mon abonnement débute avec le PROCHAIN numéro à paraître, soit :

Volume $\mathrm{N}^{\circ}$

Faites-nous parvẹitr vos coordonnées :

Nom:

Orgunisation :

Adresse :

Tetéphone :

Télécopieur :

Courriel:

Adresse d'expédition: $\quad$ Revue Organisations et territoires

Université du Québec à Chicoutimi

555, boulevard de l'Université, bureau H6-1430

Chicoutimi (Québec) CANADA G7H 2B1

Téléphone : (418) 545-5011, poste 4530 Télécopieur : (418) 693-9072

Courriel : revueot@uqac.ca SiteWeb : www.uqac.ca/revueot 